\title{
Complexity of surgery manifolds and Cheeger-Gromov invariants
}

\author{
Jae Choon Cha
}

\begin{abstract}
We present new lower bounds on the complexity of Dehn surgery manifolds of knots, using our recent result on the Cheeger-Gromov rho invariants and triangulations. As an application, we give explicit examples of closed hyperbolic 3-manifolds with fixed first homology for which the gap between the Gromov norm and the complexity is arbitrarily large.
\end{abstract}

\section{Main results}

In a recent paper Chab, we have presented explicit linear universal bounds of the Cheeger-Gromov $L^{2} \rho$-invariants of 3-manifolds in terms of topological descriptions, especially triangulations of 3-manifolds. In this paper, we use the results of Chab to study the complexity of surgery manifolds.

\section{Complexity of surgery manifolds of knots}

For a 3-manifold $M$, the complexity $c(M)$ is defined by

$$
c(M):=\min \{\text { the number of } 3 \text {-simplices in a pseudo-simplicial triangulation of } M \text { \}. }
$$

Here, a pseudo-simplicial triangulation designates a collection of disjoint 3-simplices together with affine identifications of their faces in pairs, whose quotient space is homeomorphic to the manifold. (Sometimes it is just called a triangulation.) It is more flexible than a simplicial complex structure and studied extensively in the 3-manifold literature. This definition of $c(M)$ is equivalent to Matveev's complexity Mat90 for closed irreducible 3-manifolds $M \neq S^{3}, \mathbb{R} P^{3}, L(3,1)$.

The determination of $c(M)$ is regarded as a hard problem [JRT13. In particular the main difficulty is in finding an efficient lower bound.

As the first result of this paper, we give new lower bounds for the complexity for Dehn surgery manifolds of knots, which are strong enough to determine the complexity asymptotically.

To state it, we use the following notations. For a knot $K$ in $S^{3}$ and $n \in \mathbb{Z}$, let $M(K, n)$ be the 3-manifold obtained by Dehn surgery on $K$ with slope $n$. We denote by $c(K)$ the crossing number of $K$, that is, the minimal number of crossings in a planar diagram of $K$. For two functions $f(n)$ and $g(n)$, we write $f(n) \in \Theta(g(n))$ if

$$
0<\liminf _{n \rightarrow \infty} \frac{|f(n)|}{|g(n)|} \text { and } \limsup _{n \rightarrow \infty} \frac{|f(n)|}{|g(n)|}<\infty
$$

that is, the asymptotic growth of $f(n)$ and $g(n)$ are equivalent. In terms of the big $O$ notation, $f(n) \in \Theta(g(n))$ if and only if $f(n) \in O(g(n))$ and $g(n) \in O(f(n))$.

Theorem A. For any knot $K$ in $S^{3}$ and any integer $n \neq 0$,

$$
\begin{gathered}
\frac{|n|-3-6 c(K)}{627419520} \leq c(M(K, n)) \leq 96|n|+128 c(K) . \\
\text { Consequently, } c(M(K, n)) \in \Theta(n) \text {, and } \lim _{|n| \rightarrow \infty} c(M(K, n))=\infty .
\end{gathered}
$$


The upper bound for $c(M(K, n))$ in Theorem $\mathrm{A}$ is an immediate consequence of [Chaa, Theorem A]. We give a proof of the lower bound in Section 2, using results on the Cheeger-Gromov invariants and triangulations in Chab.

We remark that while the statement of Theorem $\mathrm{A}$ (and Theorem $\mathrm{B}$ stated below) is purely 3-dimensional, its proof, including the theory developed in [Chab], is essentially 4-dimensional.

We remark that in Chab a lower bound

$$
\frac{|n|-3}{627419520} \leq c(L(n, 1)) .
$$

for the lens spaces $L(n, 1)$ was obtained, and was shown to be arbitrarily larger than lower bounds from previously known methods for any large odd $n$. Our Theorem $\AA$ subsumes (1.1) as a special case.

Note that the lower bound in Theorem $\mathrm{A}$ is useful when the surgery slope $n$ is large. Our method also presents another lower bound, which is given in terms of classical signature invariants of the given knot $K$. To state this result, we need the following notation. Recall that for a knot $K$ in $S^{3}$, a Seifert matrix $A$ is defined by choosing a Seifert surface $F$ and a basis of $H_{1}(F)$. The Levine-Tristram signature function of $K$ is defined by

$$
\sigma_{K}(\omega):=\operatorname{sign}\left((1-\omega) A+(1-\bar{\omega}) A^{T}\right), \quad \omega \in S^{1} \subset \mathbb{C} .
$$

We denote the average of the signature function over the $d$ th roots of unity by

$$
\bar{\sigma}(L, d):=\frac{1}{d} \sum_{k=1}^{d-1} \sigma_{L}\left(e^{2 \pi k \sqrt{-1} / d}\right) .
$$

Theorem B. For any knot $K$ in $S^{3}$ and for any integer $n \neq 0$,

$$
c(M(K, n)) \geq \frac{3|\bar{\sigma}(K,|n|)|-|n|+1}{627419520} .
$$

In what follows we discuss an application.

\section{Gromov norm, stable complexity, and complexity}

Recall that the Gromov norm (or simplicial volume) of a closed orientable manifold $M$ is defined by

$$
\|M\|:=\inf \{\|c\|: c \text { is a real singular cycle representing the fundamental class of } M\}
$$

where $\|c\|:=\sum\left|a_{\sigma}\right|$ denotes the $L^{1}$-norm of a chain $c=\sum_{\sigma} a_{\sigma} \sigma$ Gro82. Due to Gromov and Thurston, if $M$ is a closed hyperbolic 3-manifold, then $\|M\|=\operatorname{Vol}(M) / v_{3}$ where $v_{3}=1.01494 \cdots$ is the volume of a regular ideal tetrahedron in $\mathbb{H}^{3}$ [Thu78].

Since we allow more flexible simplices for the Gromov norm when it is compared with the complexity, we obtain immediately the following (see also [MPV09, Proposition 2.1]):

$$
\|M\| \leq c(M) .
$$

Using Thurston's hyperbolic Dehn surgery theorem, it can be observed that the gap $c(M)-\|M\|$ of (1.2) can be large: for any hyperbolic knot, Dehn surgery along large slopes gives infinitely many hyperbolic 3 -manifolds with bounded Gromov norm. Since there are only finitely many 3 -manifolds with fixed complexity, it follows that these Dehn surgery manifolds have unbounded complexity. Note that this does not give us explicit examples with large gap nor any estimate of the gap $c(M)-\|M\|$. 
We remark that in spite of this the inequality (1.2) has been used as a practically useful lower bound for $c(M)$ for hyperbolic 3-manifolds in the literature, implicitly expecting that (1.2) is not far from optimal in the hyperbolic case. For instance see MPV09, PV09.

Using our method, we give explicit examples of closed hyperbolic 3-manifolds $M$ with fixed first homology, for which the gap $c(M)-\|M\|$ is estimated to be large. Let $J_{n}$ be the 2-bridge knot in Figure 1 .

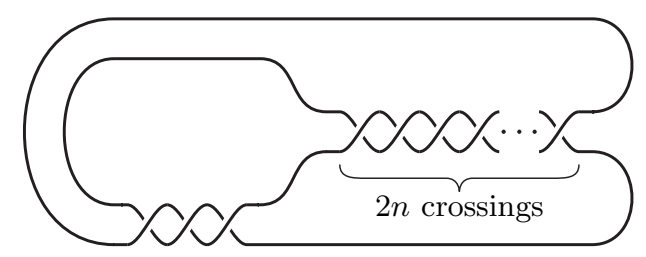

FiguRE 1. A 2-bridge knot $J_{n}$

Theorem C. Suppose $d>1$ is a fixed integer, and let $M_{n}=M\left(J_{n}, d\right)$. Then for any $n>2, M_{n}$ is hyperbolic and

$$
\begin{aligned}
& \qquad\left(M_{n}\right)-\left\|M_{n}\right\|>\frac{1}{627419520}\left(3\left(1-\frac{1}{d^{2}}\right) n-(d+7)\right)-6 . \\
& \text { Consequently } \lim _{n \rightarrow \infty} c\left(M_{n}\right)-\left\|M_{n}\right\|=\infty .
\end{aligned}
$$

The proof of Theorem $\mathrm{C}$ relies on Theorem B. the $2 \pi$-theorem of Thurston and Gromov, and computer-assisted computation using SnapPy [CDW]. For details, see Section 3 .

Note that $H_{1}\left(M_{n}\right)=\mathbb{Z}_{d}$ is fixed for the 3-manifolds $M_{n}$ in Theorem $\mathrm{C}$. We remark that for odd $d$, it follows that previously known lower bounds do not detect the divergence of $c\left(M_{n}\right)$. Specifically, the lower bounds for $c\left(M_{n}\right)$ obtained from the $\mathbb{Z}_{2}$-Thurston norm (and the double cover) in JRT09, JRT11, JRT13 vanish. Also, the lower bound for $c\left(M_{n}\right)$ obtained from the first homology in [MP01] is a constant $\left(=\log _{5} d\right)$; especially it vanishes if $d<5$.

In their study of characteristic numbers of 3-manifolds [MT77, Milnor and Thurston introduced the notion of stable complexity, which is defined by

$$
\sigma(M)=\inf \left\{\frac{c(\tilde{M})}{k} \mid \tilde{M} \rightarrow M \text { is a finite cover of degree } k\right\} .
$$

It is known that $\|M\| \leq \sigma(M) \leq c(M)$ (e.g., see [FFM12]). By Francaviglia, Frigerio, and Martelli [FFM12, Proposition 1.6], there is a constant $D_{3}$ such that $\sigma(M) \leq D_{3} \cdot\|M\|$ for any closed hyperbolic 3-manifold $M$. From this and Theorem $\mathrm{C}$, we immediately obtain that the gap between the complexity and the stable complexity can be arbitrarily large for closed hyperbolic 3-manifolds. More specifically, we have:

Corollary D. If the 3-manifolds $M_{n}$ are as in Theorem $\square, \lim _{n \rightarrow \infty} c\left(M_{n}\right)-\sigma\left(M_{n}\right)=\infty$.

\section{Acknowledgements}

This work was partially supported by NRF grants 2013067043 and 2013053914.

\section{Proofs of complexity estimates}

We begin with definitions and some preparations in Sections 2.1 and 2.2. In Section 2.3. we will prove the lower bounds in Theorems $\mathrm{A}$ and $\mathrm{B}$. 


\subsection{Cheeger-Gromov rho invariants and complexity}

For a closed $(4 k-1)$-dimensional smooth manifold $M$ and a homomorphism $\phi: \pi_{1}(M) \rightarrow$ $G$, Cheeger and Gromov defined the $L^{2} \rho$-invariant $\rho^{(2)}(M, \phi) \in \mathbb{R}$ as the difference of the ordinary and von Neumann $L^{2}$ eta invariants of the odd signature operator CG85. It is known that $\rho^{(2)}(M, \phi)$ can be defined as the $L^{2}$-signature defect of an appropriate bounding $4 k$-manifold. (This also enables us to define $\rho^{(2)}(M, \phi)$ for topological manifolds.)

For the use in Section 2.2, we describe the $L^{2}$-signature defect definition of $\rho^{(2)}(M, \phi)$ for 3-manifolds. Given $\phi: \pi_{1}(M) \rightarrow G$, it is known that there is an embedding of $G$ into another group $\Gamma$ and a 4-manifold $W$ bounded by $M$ for which there is a commutative diagram as follows:

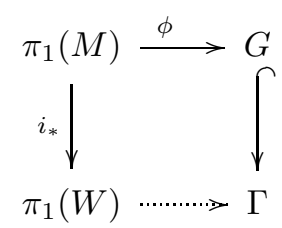

In fact, by Kan and Thurston [KT76, any $G$ embeds into an acyclic group $\Gamma$, and by bordism theory, such $W$ exists whenever $\Gamma$ is acyclic.

We consider the homology $H_{*}(W ; \mathcal{N} \Gamma)$ of $W$ with coefficients in the group von Neumann algebra $\mathcal{N} \Gamma$ of $\Gamma$, which is an algebra containing the group ring $\mathbb{C} \Gamma$. By Poincaré duality, the intersection form

$$
\lambda: H_{2}(W ; \mathcal{N} \Gamma) \times H_{2}(W ; \mathcal{N} \Gamma) \longrightarrow \mathcal{N} \Gamma
$$

is defined. We invoke a couple of useful properties of $\mathcal{N} \Gamma$; first there is a spectral decomposition $H_{2}(W ; \mathcal{N} \Gamma)=V_{+} \oplus V_{-} \oplus V_{0}$, where $\lambda$ is positive definite, negative definite, and zero on $V_{+}, V_{-}$, and $V_{0}$, respectively. Second, the $L^{2}$-dimension $\operatorname{dim}_{\Gamma}^{(2)} V \in \mathbb{R} \cup\{\infty\}$ is defined for any module $V$ over $\mathcal{N} \Gamma$. In our case, it turns out that $\operatorname{dim}_{\Gamma}^{(2)} V_{ \pm}$is finite. We define the $L^{2}$-signature of $W$ over $\Gamma$ by

$$
\operatorname{sign}_{\Gamma}^{(2)}(W):=\operatorname{dim}_{\Gamma}^{(2)} V_{+}-\operatorname{dim}_{\Gamma}^{(2)} V_{-} .
$$

Now the Cheeger-Gromov invariant of $(M, \phi)$ is defined by

$$
\rho^{(2)}(M, \phi):=\operatorname{sign}_{\Gamma}^{(2)}(W)-\operatorname{sign}(W)
$$

where $\operatorname{sign}(W)$ designates the ordinary signature of $W$. It is known that $\rho^{(2)}(M, \phi)$ is independent of the choice of the diagram (2.1). For more details, the readers are referred to, for instance, Chab, Section 2.1], CW03.

Lower bounds on complexity. In CG85, Cheeger and Gromov presented a deep analytic argument which shows that for each $M$, there is a universal bound for the values of $\rho^{(2)}(M, \phi)$. That is, there is a constant $C_{M}$ such that $\left|\rho^{(2)}(M, \phi)\right| \leq C_{M}$ (for any $G$ and) for any $\phi: \pi_{1}(M) \rightarrow G$. In [Chab], we developed a topological approach to the universal bound. One of the main result was the following explicit linear bound in terms of the complexity:

Theorem 2.1 (Chab, Corollary 1.11]). If $M$ is a closed 3-manifold, then for any homomorphism $\phi$ of $\pi_{1}(M)$,

$$
\left|\rho^{(2)}(M, \phi)\right| \leq 209139840 \cdot c(M) .
$$


In the proof of Theorems $\mathrm{A}$ and $\mathrm{B}$ we will use Theorem 2.1 as a lower bound for $c(M)$. To estimate the lower bound, we will compute certain $\rho$-invariants over finite cyclic groups, using a method for general 3-manifolds discussed in the next subsection.

\subsection{Cheeger-Gromov invariants over finite cyclic groups}

In this subsection we give an explicit formula for the Cheeger-Gromov invariant of a 3 -manifold over a finite cyclic group (Theorem 2.2). In fact, in this case, $\rho^{(2)}(M, \phi)$ can be obtained from the Atiyah-Singer invariants AS68, which are essentially equivalent to the Casson-Gordon invariants [CG78. Our proof depends on results of CassonGordon CG78, and Gilmer Gil81, which in turn can be shown using the Atiyah-Singer $G$-signature theorem.

Suppose $K$ is an oriented knot in $S^{3}$ and $n$ and $r$ are integers. We call a union of finitely many disjoint parallels of $K$ an $n$-twisted $r$-cable if each parallel is taken along the $n$-framing of $K$ and oriented in such a way that the sum of the parallels is homologous to $r K$ in a tubular neighborhood of $K$.

Theorem 2.2. Suppose $M$ is a closed 3-manifold, and $L=K_{1} \sqcup \cdots \sqcup K_{r}$ is an $r$ component oriented link in $S^{3}$ such that surgery on $L$ with integral coefficients $n_{1}, \ldots, n_{r}$ gives $M$. Let $\Lambda=\left(n_{i j}\right)$ be the linking matrix defined by $n_{i i}=n_{i}$ and $n_{i j}=\operatorname{lk}\left(K_{i}, K_{j}\right)$ for $i \neq j$. Suppose $\phi: \pi_{1}(M) \rightarrow \mathbb{Z}_{d}$ is a homomorphism. Let $\mu_{i}$ be the positive meridian of $K_{i}$, and let $r_{i}$ be an integer satisfying $r_{i}=\phi\left(\mu_{i}\right)$ in $\mathbb{Z}_{d}$. Let $L^{\prime}$ be the link obtained from $L$ by replacing each component $K_{i}$ with a nonempty $n_{i}$-twisted $r_{i}$-cable of $K_{i}$. Then we have

$$
\rho^{(2)}(M, \phi)=\bar{\sigma}\left(L^{\prime}, d\right)-\frac{d-1}{d} \operatorname{sign} \Lambda+\frac{d^{2}-1}{3 d^{2}} \sum_{i, j} r_{i} r_{j} n_{i j} .
$$

Proof. In [CG86], Casson and Gordon defined an invariant $\sigma_{r}(M, \phi)$ as follows. Since $\Omega_{3}\left(\mathbb{Z}_{d}\right)$ is finite, there is a 4 -manifold $W$ over $\mathbb{Z}_{d}$ such that $\partial W=s M$ over $\mathbb{Z}_{d}$ for some integer $s \neq 0$. Let $\tilde{W}$ be the $\mathbb{Z}_{d}$-cover of $W$. The generator $1 \in \mathbb{Z}_{d}$ induces an order $d$ linear operator $g: H_{2}(\tilde{W} ; \mathbb{C}) \rightarrow H_{2}(\tilde{W} ; \mathbb{C})$. Let $\sigma_{k}(\tilde{W})$ be the signature of the intersection form of $\tilde{W}$ restricted on the $e^{2 \pi k \sqrt{-1} / d}$-eigenspace of $g$. Then the rational number

$$
\sigma_{k}(M, \phi):=\frac{1}{s}\left(\sigma_{k}(\tilde{W})-\operatorname{sign}(W)\right)
$$

is well-defined, independent of the choice of $W$. (Our sign convention is opposite of that of [CG86 but agrees with that of Gil81.) It is known that $\sigma_{0}(M, \phi)=0$; e.g., see [CG86, p. 40]. Due to Gilmer [Gil81, Theorem 3.6], we have

$$
\sigma_{k}(M, \phi)=\sigma_{L^{\prime}}\left(e^{2 \pi k \sqrt{-1} / d}\right)-\operatorname{sign} \Lambda+\frac{2(d-k) k}{d^{2}} \sum_{i, j} r_{i} r_{j} n_{i j}
$$

for $0<k<d$. See also CG78, Section 3] for a special case.

It is related to $\rho^{(2)}(M, \phi)$ as follows. Since $\mathbb{Z}_{d}$ is finite, the group von Neumann algebra $\mathcal{N} \mathbb{Z}_{d}$ is equal to the ordinary group ring $\mathbb{C}\left[\mathbb{Z}_{d}\right]$ and the $L^{2}$-dimension over $\mathbb{Z}_{d}$ is given by $\operatorname{dim}_{\mathbb{Z}_{d}}^{(2)} V=\frac{1}{d} \operatorname{dim}_{\mathbb{C}} V$. Observe that $H_{2}\left(W ; \mathcal{N}_{\mathbb{Z}}\right)=H_{2}\left(W ; \mathbb{C}\left[\mathbb{Z}_{d}\right]\right) \cong H_{2}(\tilde{W} ; \mathbb{C})$ is the orthogonal sum of the $e^{2 \pi k \sqrt{-1} / d}$-eigenspaces $(k=0, \ldots, d-1)$. From the definitions of $\sigma_{k}(\tilde{W})$ and $\operatorname{sign}_{\mathbb{Z}_{d}}^{(2)}(W)$ (see (2.2) in Section 2.1), it follows that

$$
\operatorname{sign}_{\mathbb{Z}_{d}}^{(2)} W=\frac{1}{d} \sum_{k=0}^{d-1} \sigma_{k}(\tilde{W}) .
$$


From this and (2.3), it follows that

$$
\rho^{(2)}(M, \phi)=\frac{1}{s}\left(\operatorname{sign}_{\mathbb{Z}_{d}}^{(2)} W-\operatorname{sign} W\right)=\frac{1}{d} \sum_{k=0}^{d-1} \sigma_{k}(M, \phi) .
$$

Substituting (2.4) into (2.5), we obtain the desired formula for $\rho^{(2)}(M, \phi)$.

Using Theorem 2.2, we give an explicit formula for the Cheeger-Gromov invariant of surgery manifolds of knots.

Corollary 2.3. Suppose $K$ is a knot in $S^{3}, n>0$, and let

$$
\phi: \pi_{1}(M(K, n)) \longrightarrow H_{1}(M(K, n))=\mathbb{Z}_{n}
$$

be the abelianization. Then

$$
\rho^{(2)}(M(K, n), \phi)=\frac{n}{3}+\frac{2}{3 n}-1+\bar{\sigma}(K, n) .
$$

Proof. Note that $\phi$ takes a meridian of $K$ to $1 \in \mathbb{Z}_{n}$. Also, the $n$-twisted 1-cable of $K$ is $K$ itself, and the linking matrix for the $n$-surgery on $K$ is $\Lambda=[n]$. Therefore, by applying Theorem 2.2, we obtain the formula for $\rho^{(2)}(M(K, n), \phi)$.

\subsection{Lower bounds of the complexity of surgery manifolds of knots}

For a knot $K$ in $S^{3}$, we denote by $g_{4}(K)$ the (topological) slice genus of $K$. That is, $g_{4}(K)$ is the minimal genus of a properly embedded locally flat orientable surface in $B^{4}$ bounded by $K$.

Theorem 2.4. Suppose $K$ is a knot in $S^{3}$, and $n \neq 0$. Then

$$
c(M(K, n)) \geq \frac{|n|-3-6 g_{4}(K)}{627419520} .
$$

The slice genus $g_{4}(K)$ in Theorem 2.4 can be replaced by either one of the smooth slice genus, the unknotting number, the Seifert genus, or the crossing number of $K$, since $g_{4}(K)$ is not greater than any one of them. In particular, the lower bound part of Theorem A in the introduction is an immediate consequence of Theorem 2.4.

Proof of Theorem 2.4. We may assume that $n>0$, by taking the mirror image of $K$ if $n<0$. Let $\phi: \pi_{1}(M(K, n)) \rightarrow \mathbb{Z}_{n}$ be the abelianization. From Corollary 2.3, we obtain

$$
\left|\rho^{(2)}(M(K, n), \phi)\right| \geq \frac{n-3-3|\bar{\sigma}(K, n)|}{3} .
$$

It is known that $\left|\sigma_{K}(\omega)\right| \leq 2 g_{4}(K)$ for any root of unity $\omega \in S^{1}$ (for example, see [Tay79, p. 145]). From this it follows that

$$
|\bar{\sigma}(K, n)| \leq \frac{1}{n} \sum_{k=0}^{n-1}\left|\sigma_{K}\left(e^{2 \pi k \sqrt{-1}}\right)\right| \leq 2 g_{4}(K) .
$$

From (2.6) and (2.7), it follows that

$$
\left|\rho^{(2)}(M(K, n), \phi)\right| \geq \frac{n-3-6 g_{4}(K)}{3} .
$$

By combining Theorem 2.1 and (2.8), we obtain the inequality. 
Proof of Theorem $B$. Similarly to the above proof of Theorem 2.4 we may assume $n>0$. Then it is easily verified that $\frac{n}{3}+\frac{2}{3 n}-1$ is nonnegative. From this and from Corollary 2.3 . it follows that

$$
\begin{aligned}
\left|\rho^{(2)}(M(K, n), \phi)\right| & \geq|\bar{\sigma}(K, n)|-\left(\frac{n}{3}+\frac{2}{3 n}-1\right) \\
& \geq \frac{3|\bar{\sigma}(K, n)|-n+1}{3} .
\end{aligned}
$$

By combining Theorem 2.1 and (2.9), we obtain

$$
c(M(K, n)) \geq \frac{3|\bar{\sigma}(K, n)|-n+1}{627419520} .
$$

\section{Computation for examples}

This section is devoted to the proof of Theorem C. In the first subsection we show that the surgery manifolds considered in Theorem C are hyperbolic using Gromov-Thurston's $2 \pi$-theorem, and estimate the Gromov norm. In the second subsection we estimate the complexity of $M\left(J_{n}, d\right)$ via computation of signature invariants, using Theorem B.

\subsection{Hyperbolicity and Gromov norm}

Consider the link $L$ shown in Figure 2

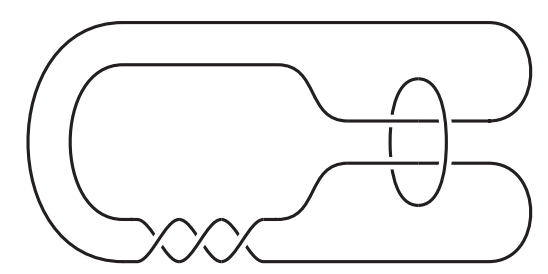

FiguRE 2. A two-component hyperbolic link $L$ with volume 5.3335

According to computation using SnapPy [CDW], $L$ is a hyperbolic link with $\operatorname{Vol}\left(S^{3}\right.$ $L)=5.3335$. Also, for both cusps, the translation lengths of the meridian and the longitude (on the boundary of a maximal horoball neighborhood) are $-0.4204+1.1124 \sqrt{-1}$ and 3.3636, respectively.

It follows that the length of the slope $k \in \mathbb{Z}$ is greater than 6.7271 if $k>5$, and the length of the slope $1 / n$ with $n \in \mathbb{Z}$ is greater than 6.4040 if $n>1$. By the $2 \pi$-theorem of Gromov and Thurston and by the geometrization conjecture, it follows that the $(k, 1 / n)$ surgery on the link $L$ is hyperbolic if $k>5$ and $n>1$, and by the hyperbolic Dehn surgery theorem, its Gromov norm is bounded by $\operatorname{Vol}\left(S^{3} \backslash L\right) / v_{3}=5.2552$.

Observe that the $(1 / n)$-surgery on the component drawn as a circle in Figure 2 introduces $n$ left-handed full twists between the two enclosed strands, so that the other component becomes the 2-bridge knot $J_{n}$ shown in Figure 1 . Also, the $(1 / n)$-surgery alters the framing of the other component by $-4 n$ (for this we use that the two enclosed strands have the same orientation). It follows that the surgery manifold $M\left(J_{n}, d\right)$ is equal to the $(d+4 n, 1 / n)$-surgery on the link $L$.

Summarizing, we have shown the following: $M\left(J_{n}, d\right)$ is hyperbolic with $\left\|M\left(J_{n}, d\right)\right\|<$ 5.2552 for any $d>1$ and $n>1$. 


\subsection{Complexity}

To obtain a lower bound for the complexity of $M\left(J_{n}, d\right)$ using Theorem B, we need to compute the Levine-Tristram signature function of the 2-bridge knot $J_{n}$. Using the Seifert surface and the generators $x_{i}$ shown in Figure 3, we obtain a $2 n \times 2 n$ Seifert matrix $A$ for $J_{n}$ :

$$
A=\left[\begin{array}{ccccc}
1 & 1 & & & \\
& 1 & 1 & & \\
& & \ddots & \ddots & \\
& & & 1 & 1 \\
& & & & -1
\end{array}\right] .
$$

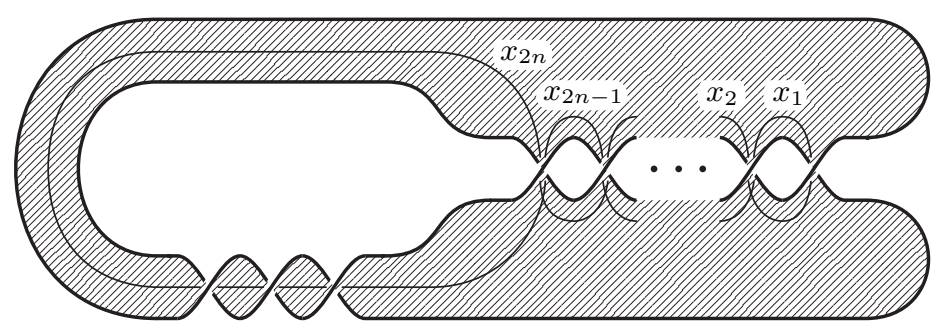

Figure 3. A Seifert surface for the 2-bridge knot $J_{n}$.

Observe that if we replace the bottom-right entry -1 by $1, A$ becomes the Seifert matrix for the $(2,2 n+1)$ torus knot $T_{2,2 n+1}$ shown in Figure 4 .

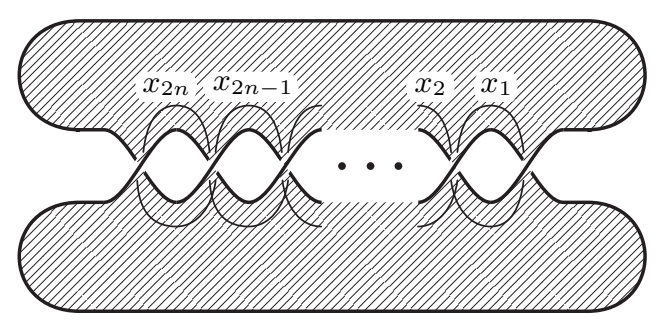

FIgURE 4 . The torus knot $T_{2,2 n+1}$ with a Seifert surface.

It follows that the Levine-Tristram signatures for $J_{n}$ and $T_{2,2 n+1}$ differ by at most two, and consequently so do their averages: for any $d$,

$$
\left|\bar{\sigma}\left(J_{n}, d\right)-\bar{\sigma}\left(T_{2,2 n+1}, d\right)\right| \leq 2 .
$$

By work of Litherland Lit79, the Levine-Tristram signature function of a torus knot is well-understood. Especially, for the case of $T_{2,2 n+1}$, Proposition 1 of [Lit79] gives us

$$
\sigma_{T_{2,2 n+1}}\left(e^{2 \pi x \sqrt{-1}}\right)=2 n-2\left\lfloor(2 n+1)\left(\frac{1}{2}-x\right)\right\rfloor
$$

for rational $x \in\left(0, \frac{1}{2}\right]$. It follows that

$$
\begin{aligned}
\bar{\sigma}\left(T_{2,2 n+1}, d\right) & \geq \frac{2}{d} \sum_{k=1}^{(d-1) / 2}\left(2 n-2 \cdot(2 n+1)\left(\frac{1}{2}-\frac{k}{d}\right)\right) \\
& =\left(1-\frac{1}{d^{2}}\right) n-\frac{(d-1)^{2}}{2 d^{2}}
\end{aligned}
$$


for odd $d$, and

$$
\begin{aligned}
\bar{\sigma}\left(T_{2,2 n+1}, d\right) & \geq \frac{1}{d}\left(2 \sum_{k=1}^{(d / 2)-1}\left(2 n-2 \cdot(2 n+1)\left(\frac{1}{2}-\frac{k}{d}\right)\right)+2 n\right) \\
& =n-\frac{d-2}{2 d}
\end{aligned}
$$

for even $d$. In both cases, we have

$$
\bar{\sigma}\left(T_{2,2 n+1}, d\right) \geq\left(1-\frac{1}{d^{2}}\right) n-\frac{d-1}{2 d} .
$$

From (3.1) and (3.2), we obtain

$$
\bar{\sigma}\left(J_{n}, d\right) \geq\left(1-\frac{1}{d^{2}}\right) n-\frac{5 d-1}{2 d}
$$

where the right hand side is positive for $n \geq 3, d \geq 2$. Now, combining (3.3) and Theorem B, we obtain

$$
\begin{aligned}
c\left(M_{n}\right) & \geq \frac{1}{627419520}\left(3\left(1-\frac{1}{d^{2}}\right) n-\frac{15 d-3}{2 d}-d+1\right) \\
& \geq \frac{1}{627419520}\left(3\left(1-\frac{1}{d^{2}}\right) n-(d+7)\right)
\end{aligned}
$$

Together with the last sentence of Section 3.1, it completes the proof of Theorem C

\section{References}

[AS68] M. F. Atiyah and I. M. Singer, The index of elliptic operators. III, Ann. of Math. (2) 87 (1968), 546-604.

[CDW] Marc Culler, Nathan M. Dunfield, and Jeffrey R. Weeks, SnapPy, a computer program for studying the topology of 3-manifolds, Available at http://snappy.computop.org

[CG78] Andrew Casson and Cameron Gordon, On slice knots in dimension three, Algebraic and geometric topology (Proc. Sympos. Pure Math., Stanford Univ., Stanford, Calif., 1976), Part 2, Amer. Math. Soc., Providence, R.I., 1978, pp. 39-53.

[CG85] Jeff Cheeger and Mikhael Gromov, Bounds on the von Neumann dimension of L ${ }^{2}$-cohomology and the Gauss-Bonnet theorem for open manifolds, J. Differential Geom. 21 (1985), no. 1, 134.

[CG86] Andrew Casson and Cameron Gordon, Cobordism of classical knots, À la recherche de la topologie perdue, Birkhäuser Boston, Boston, MA, 1986, With an appendix by P. M. Gilmer, pp. 181-199.

[Chaa] Jae Choon Cha, Complexities of 3-manifolds from triangulations, heegaard splittings, and surgery presentations, Preprint.

[Chab] _ A topological approach to Cheeger-Gromov universal bounds for von neumann rhoinvariants, arXiv:1405.1805, to appear in Comm. Pure Appl. Math.

[CW03] Stanley Chang and Shmuel Weinberger, On invariants of Hirzebruch and Cheeger-Gromov, Geom. Topol. 7 (2003), 311-319 (electronic).

[FFM12] Stefano Francaviglia, Roberto Frigerio, and Bruno Martelli, Stable complexity and simplicial volume of manifolds, J. Topol. 5 (2012), no. 4, 977-1010.

[Gil81] Patrick M. Gilmer, Configurations of surfaces in 4-manifolds, Trans. Amer. Math. Soc. 264 (1981), no. 2, 353-380.

[Gro82] Michael Gromov, Volume and bounded cohomology, Inst. Hautes Études Sci. Publ. Math. (1982), no. 56, 5-99 (1983).

[JRT09] William Jaco, Hyam Rubinstein, and Stephan Tillmann, Minimal triangulations for an infinite family of lens spaces, J. Topol. 2 (2009), no. 1, 157-180.

[JRT11] William Jaco, J. Hyam Rubinstein, and Stephan Tillmann, Coverings and minimal triangulations of 3-manifolds, Algebr. Geom. Topol. 11 (2011), no. 3, 1257-1265.

[JRT13] — $\mathbb{Z}_{2}$-Thurston norm and complexity of 3-manifolds, Math. Ann. 356 (2013), no. 1, $1-22$. 
[KT76] D. M. Kan and W. P. Thurston, Every connected space has the homology of a $K(\pi, 1)$, Topology 15 (1976), no. 3, 253-258.

[Lit79] R. A. Litherland, Signatures of iterated torus knots, Topology of low-dimensional manifolds (Proc. Second Sussex Conf., Chelwood Gate, 1977), Springer, Berlin, 1979, pp. 71-84.

[Mat90] S. V. Matveev, Complexity theory of three-dimensional manifolds, Acta Appl. Math. 19 (1990), no. $2,101-130$.

[MP01] S. V. Matveev and E. L. Pervova, Lower bounds for the complexity of three-dimensional manifolds, Dokl. Akad. Nauk 378 (2001), no. 2, 151-152.

[MPV09] Sergei Matveev, Carlo Petronio, and Andrei Vesnin, Two-sided asymptotic bounds for the complexity of some closed hyperbolic three-manifolds, J. Aust. Math. Soc. 86 (2009), no. 2, 205-219.

[MT77] J. Milnor and W. Thurston, Characteristic numbers of 3-manifolds, Enseignement Math. (2) 23 (1977), no. 3-4, 249-254.

[PV09] Carlo Petronio and Andrei Vesnin, Two-sided bounds for the complexity of cyclic branched coverings of two-bridge links, Osaka J. Math. 46 (2009), no. 4, 1077-1095.

[Tay79] Laurence R. Taylor, On the genera of knots, Topology of low-dimensional manifolds (Proc. Second Sussex Conf., Chelwood Gate, 1977), Lecture Notes in Math., vol. 722, Springer, Berlin, 1979, pp. 144-154.

[Thu78] William Thurston, The geometry and topology of 3-manifolds, Princeton University, 1978, Lecture notes.

Department of Mathematics, POSTECH, Pohang 790-784, Republic of Korea - and School of Mathematics, Korea Institute for Advanced Study, Seoul 130-722, Republic of Korea E-mail address: jccha@postech.ac.kr 